\title{
Quasi-periodic flares in EXO 2030+375 observed with INTEGRAL
}

\author{
D. Klochkov ${ }^{1}$, C. Ferrigno ${ }^{2}$, A. Santangelo ${ }^{1}$, R. Staubert ${ }^{1}$, P. Kretschmar ${ }^{3}$, I. Caballero ${ }^{4}$, \\ K. Postnov ${ }^{5}$, and C. A. Wilson-Hodge ${ }^{6}$
}

\author{
${ }^{1}$ Institut für Astronomie und Astrophysik, Universität Tübingen (IAAT), Sand 1, 72076 Tübingen, Germany \\ e-mail:klochkov@astro.uni-tuebingen.de \\ 2 ISDC Data Center for Astrophysics of the University of Geneva, chemin d'Écogia 16, 1290 Versoix, Switzerland \\ 3 European Space Astronomy Centre (ESA/ESAC), Science Operations Department, Villanueva de la Canãda (Madrid), Spain \\ ${ }^{4}$ CEA Saclay, DSM/IRFU/SAp-UMR AIM (7158) CNRS/CEA/Université Paris Diderot, 91191 Gif-sur-Yvette, France \\ 5 Sternberg Astronomical Institute, Universitetski pr. 13, 119991 Moscow, Russia \\ ${ }^{6}$ NASA Marshall Space Flight Center, Huntsville, AL 35812, USA \\ Received 30 September 2011 / Accepted 6 November 2011
}

\section{ABSTRACT}

\begin{abstract}
Context. Episodic flaring activity is a common feature of X-ray pulsars in HMXBs. In some Be/X-ray binaries flares were observed in quiescence or prior to outbursts. EXO 2030+375 is a Be/X-ray binary showing "normal" outbursts almost every 46 days, near periastron passage of the orbital revolution. Some of these outbursts were occasionally monitored with the INTEGRAL observatory. Aims. The INTEGRAL data revealed strong quasi-periodic flaring activity during the rising part of one of the system's outburst. Such activity has previously been observed in EXO 2030+375 only once, in 1985 with EXOSAT. (Some indications of single flares have also been observed with other satellites.)

Methods. We present the analysis of the flaring behavior of the source based on INTEGRAL data and compare it with the flares observed in EXO 2030+375 in 1985.

Results. Based on the observational properties of the flares, we argue that the instability at the inner edge of the accretion disk is the most probable cause of the flaring activity.
\end{abstract}

Key words. stars: neutron - accretion, accretion disks - X-rays: binaries

\section{Introduction}

Accreting X-ray pulsars, mostly residing in high-mass X-ray binaries (HMXBs), often show abrupt increases in their X-ray luminosity lasting from a few tens of seconds to several hours - X-ray flares. Flaring activity is often observed on top of a slower flux variation related to X-ray outbursts or super-orbital modulation. Among the best-known "flaring" X-ray pulsars are LMC X-4 (e.g. Moon et al. 2003b, end references therein), SMC X-1 (Angelini et al. 1991; Moon et al. 2003a), Vela X-1 (Kreykenbohm et al. 2008; Fürst et al. 2010). X-ray binaries with a donor star of $\mathrm{Be}$ (or $\mathrm{Oe}$ ) spectral type (Be/X-ray binaries or BeXRB) are currently the most numerous class of HMXBs with X-ray pulsars (Liu et al. 2006), although another class of HMXB pulsars, the supergiant fast X-ray transients (SFXT), is rapidly growing (e.g. Sidoli 2011). BeXRBs are characterized by periodic or sporadic X-ray outbursts lasting from several days to several weeks when the neutron star accretes matter from the equatorial disk around the donor star (see e.g. Reig 2011, for a recent review). These systems are also known to show occasional X-ray flares (Finger et al. 1999; Reig et al. 2008; Caballero et al. 2008; Postnov et al. 2008).

Probably the most remarkable flaring activity among BeXRBs was exhibited by the $42 \mathrm{~s}$ pulsar EXO 2030+375. A series of six roughly equidistant flares with a mean period of $\sim$ four hours was observed with the EXOSAT satellite a few months after the giant outburst of the source in 1985 (Parmar et al. 1989). EXO 2030+375 is one of the most regularly monitored BeXRBs. In addition to two "major" outbursts in 1985 and 2006 with a peak X-ray luminosity of $L_{\mathrm{X}} \gtrsim 10^{38} \mathrm{erg} \mathrm{s}^{-1}$, assuming a distance of $7 \mathrm{kpc}$ (Wilson et al. 2002), the source exhibited less powerful "normal" outbursts with $L_{\mathrm{X}} \sim 10^{37} \mathrm{erg} \mathrm{s}^{-1}$ almost every orbit. The orbital period of the system is $\sim 46 \mathrm{~d}$ (Wilson et al. 2008).

Some flaring of EXO 2030+375 has been reported since the EXOSAT observations, but it has generally appeared to be a single flare during the rise of normal outbursts (Reig \& Coe 1998; Camero Arranz et al. 2005). Flares were also apparently present during and between the normal outbursts shortly after the 2006 major outburst (Fig. 3 in Wilson et al. 2008). But the short duration and broad spacing of the observations make it unclear whether this is the same quasi-periodic phenomenon that was seen with EXOSAT. In this Letter we present the INTEGRAL observations of the source that, for the first time since the EXOSAT observations, reveal strong quasi-periodic flaring behavior.

\section{Observations and data processing}

Since its major outburst in 2006, EXO 2030+375 has repeatedly appeared in the field of view of INTErnational Gamma Ray Astrophysics Laboratory (INTEGRAL, Winkler et al. 2003) during the observational program concentrated on the "Cygnus Region". The INTEGRAL observatory has three main X-ray instruments: (i) the imager IBIS sensitive from $\sim 20 \mathrm{keV}$ to a few $\mathrm{MeV}$ with high spatial and moderate spectral resolution (Ubertini et al. 2003); (ii) the spectrometer SPI, which is sensitive in the roughly same energy range as IBIS, but with much higher spectral resolution and substantially lower imaging capability (Vedrenne et al. 2003); and (iii) the X-ray monitor 


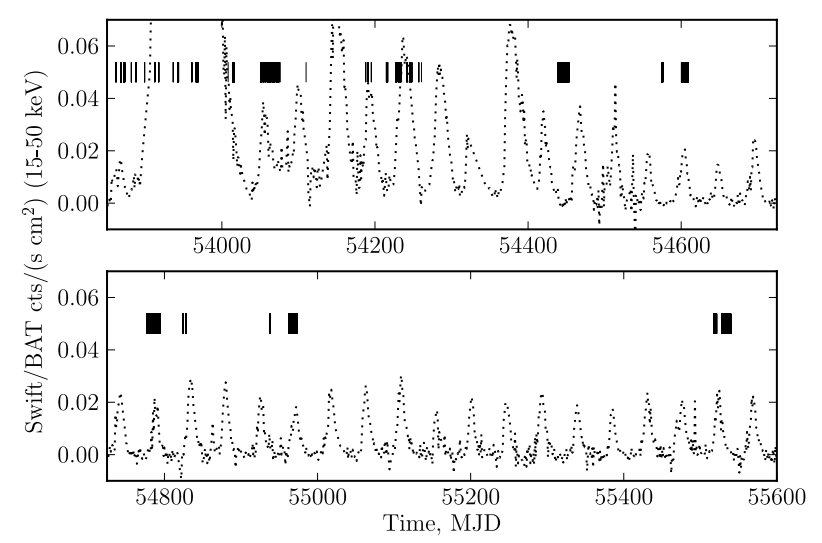

Fig. 1. INTEGRAL observations of EXO 2030+375 (vertical bars) superposed on the Swift/BAT light curve of the source since its major outburst in June-October 2006.

JEM-X with moderate spectral and spatial resolution, operating between $\sim 3$ and $\sim 35 \mathrm{keV}$ (Lund et al. 2003). INTEGRAL observations normally consist of a series of pointings called Science Windows, 2 to $4 \mathrm{ks}$ each.

Because the times of the observations were not optimized for EXO 2030+375, the INTEGRAL coverage of the source's normal outbursts is rather sparse. The Swift/BAT light curve of the pulsar ${ }^{1}$ with the indicated INTEGRAL observations is shown in Fig. 1.

For our analysis we used the ISGRI detector layer of IBIS sensitive in the $20-300 \mathrm{keV}$ energy range (Lebrun et al. 2003) and JEM-X. Owing to limited count-rate statistics, no additional information could be gained from SPI data. The standard data processing was performed with version 9 of the Offline Science Analysis (OSA) software provided by the INTEGRAL Science Data Centre (ISDC, Courvoisier et al. 2003). We performed an additional gain correction of the ISGRI energy scale based on the background Tungsten spectral lines.

To search for the flaring activity, we examined the entire IBIS/ISGRI light curve by combining the publicly available ISGRI data products in the HEAVENS data base ${ }^{2}$ with the results of our own analysis. We did not find any clear evidence of flares in all the data except for the latest INTEGRAL observations of the source in November and December 2010 (MJD $\sim 55$ 520-55 540), partially covering a normal outburst. The rising part of the outburst is shown in Fig. 2. The upper panel of Fig. 3 shows the entire outburst as observed with INTEGRAL. In this work we concentrate on the analysis of the source's flaring behavior during this outburst.

\section{Timing analysis}

As can be seen in Fig. 2, the flux in the rising part of the outburst experiences quasi-periodic oscillations/flares that cease as the averaged flux increases. One can identify at least five subsequent flares with a mean period of $\sim 0.3$ days $(\sim 7 \mathrm{~h})$.

To characterize the level of the flux variability during the outburst we calculated the normalized excess variance in the light curve in relatively broad time intervals. We defined four equal adjacent intervals in the rising part and five equal adjacent intervals in the decay. The normalized excess variance is often used

\footnotetext{
1 We used the Swift/BAT transient monitor results provided by the Swift/BAT team

${ }^{2}$ http://www.isdc.unige.ch/heavens/heavens
}



Fig. 2. INTEGRAL/ISGRI light curve of EXO 2030+375 during the rise of its normal outburst in Nov.-Dec. 2010. The flaring activity is clearly seen. The light curve of the entire outburst is shown in Fig. 3. One Crab corresponds to $\sim 260 \mathrm{cts} / \mathrm{s}$ in the specified energy range.

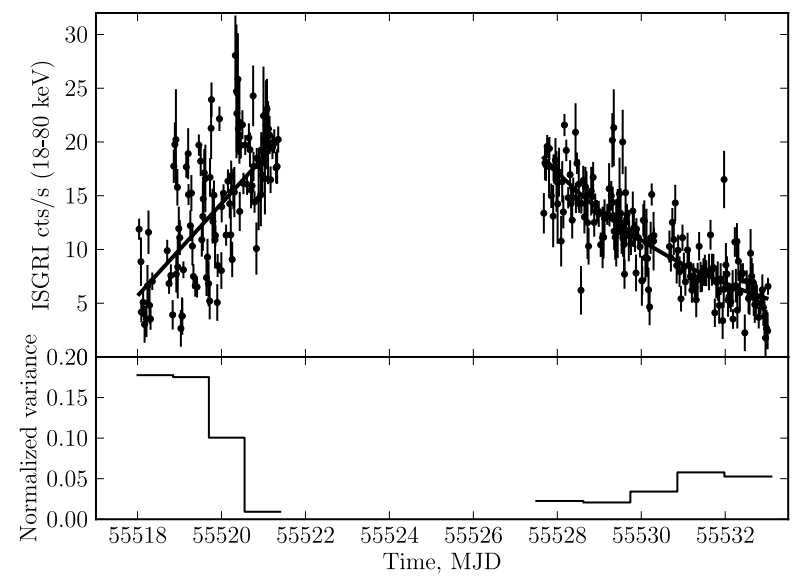

Fig. 3. Top: ISGRI light curve of the EXO 2030+375 outburst approximated with a polynomial function (solid curve) which represents the averaged evolution of the flux. Bottom: normalized excess variance of the source flux with respect to the polynomial function.

as a simple measure of the intrinsic variability amplitude in light curves, (see e.g. Nandra et al. 1997):

$\sigma_{\mathrm{NXS}}^{2}=\frac{1}{N\langle f\rangle^{2}} \Sigma_{i=1}^{N}\left[\left(f_{i}-f_{i}^{\mathrm{aver}}\right)^{2}-\sigma_{i}^{2}\right]$

Here $N$ is the number of data points in the corresponding time interval, $f_{i}$ is the flux of the individual data points, $\sigma_{i}-$ their uncertainty, $f_{i}^{\text {aver }}$ is the smoothed evolution of the flux obtained by a polynomial fit to the light curve (upper panel of Fig. 3), and $\langle f\rangle$ is the mean value of the flux within the interval. The normalized variance $\sigma_{\text {NXS }}^{2}$ in our case represents the amplitude of intrinsic flux variations superimposed on the smoothed flux development. The term $\sigma_{i}$ under the summation $(\Sigma)$ eliminates the contribution of the Poisson noise. The bottom panel of Fig. 3 represents the normalized variance as a function of time during the outburst of EXO 2030+375. It can be seen that the amplitude of the variability is high at the rising phase (corresponding to the flaring episode). Then it decreases towards the maximum of the outburst and remains low during the decay.

To study periodicity of the flares, we performed a formal period search in the rising part of the outburst (between MJD 55518.5 and 55 521.5) using the Lomb-Scargle periodogram. The results are presented in Fig. 4. The periodogram shown in the top panel indicates a clear peak around $\sim 0.3$ days $(\sim 7 \mathrm{~h})$. The averaged profile obtained by folding the light curve with this period is shown in the bottom panel. The profile shape is asymmetric and characterized by a steep rise and a slower decay. 

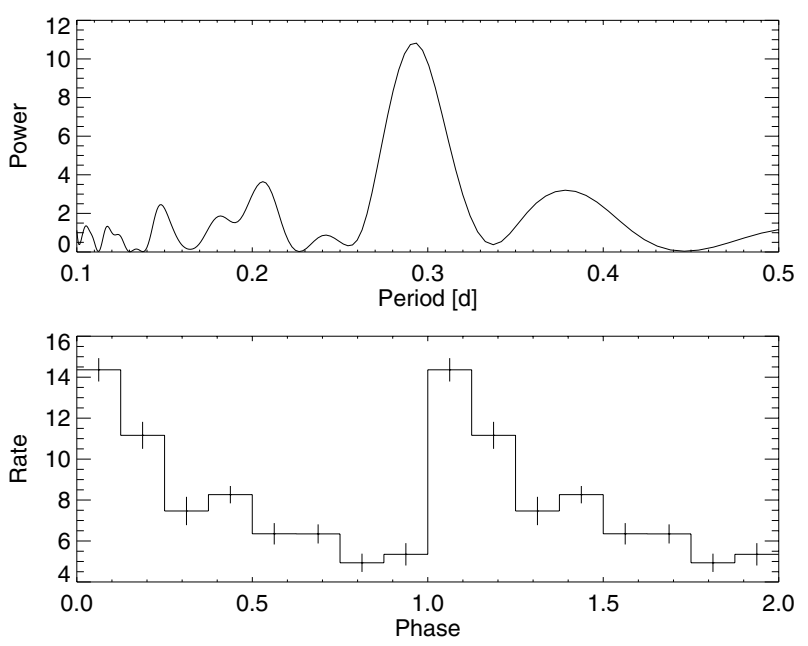

Fig. 4. Top: Lomb-Scargle periodogram of the "flaring" part of EXO 2030+375 light curve (between MJD 55518.5 and 55 521.5). The peak around $\sim 0.3 \mathrm{~d}$ is clearly seen. Bottom: "Flaring" part of the light curve folded with best period found from the periodogram $(0.293 \mathrm{~d})$.

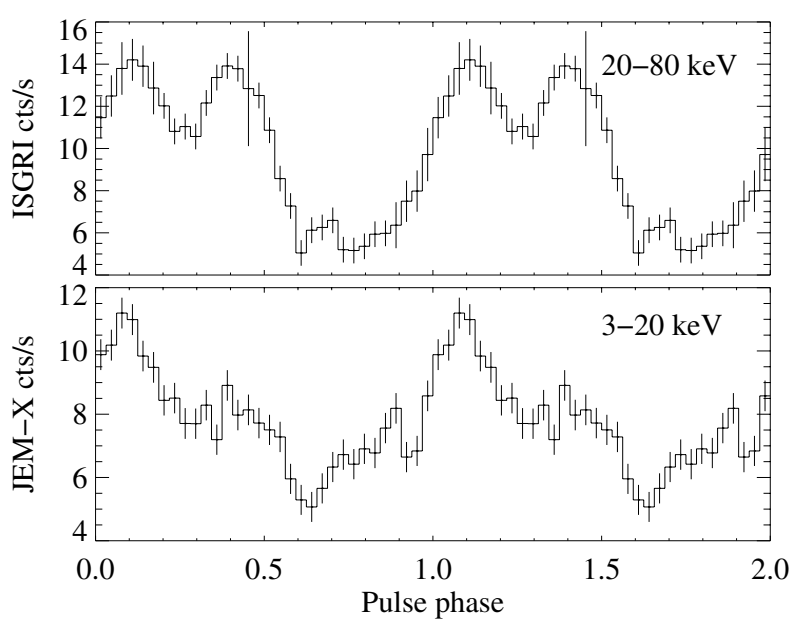

Fig. 5. Pulse profiles of EXO2030+375 obtained with ISGRI (top) and JEM-X (bottom) instruments during the outburst (MJD 55518.5 55 533.0).

We used the INTEGRAL data to study the pulse period behavior and pulse profiles during the outburst. The photon arrival times were converted to the reference frame of the solar system barycenter and corrected for the binary orbital motion using the ephemeris by Wilson et al. (2008). Using the pulsephase-connection technique (e.g. Staubert et al. 2009), we found the pulse period $P=41.31516(2) \mathrm{s}$ at the epoch $T_{0}(\mathrm{MJD})=$ 55526.056994 and the period derivative $\dot{P}=-1.9(1) \times 10^{-9} \mathrm{~s} / \mathrm{s}$, that indicates significant spin-up. We used the measured pulse ephemeris to construct and study the pulse profiles of the source. We could not find any difference between the profiles obtained during the rise and decay of the outburst. Figure 5 shows the profiles accumulated during the entire outburst.

\section{Spectral analysis}

For the spectral analysis we used JEM-X data between 3.5 and $35 \mathrm{keV}$, and ISGRI data between 20 and $80 \mathrm{keV}$. We added systematic uncertainties at the level of $2 \%$ to the JEM-X spectra and $1 \%$ to the ISGRI spectra based on the recommendations of the instrument teams and the Crab observations. The spectrum of the source during the outburst (accumulated between MJD

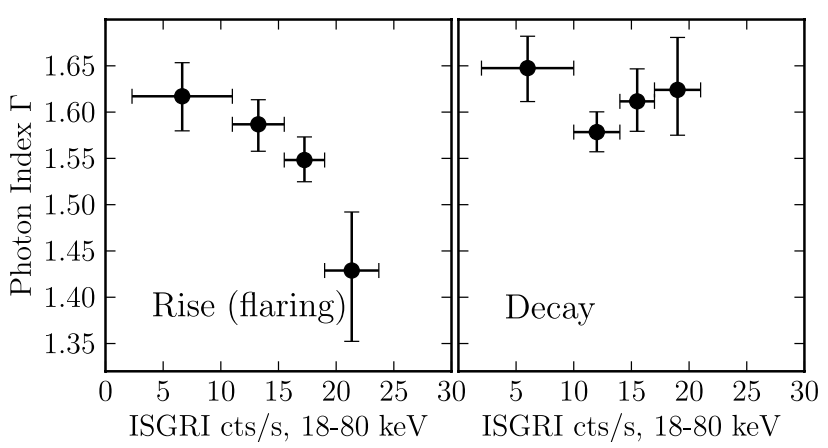

Fig. 6. Photon index $\Gamma$ as a function of flux during the rising (flaring) part of the outburst (left) and the decay phase (right). One Crab corresponds to $\sim 260 \mathrm{cts} / \mathrm{s}$ in the specified energy range.

55518.5 and 55533.0$)$ was modeled with the cutoff-powerlaw model $\left(F(E) \propto E^{-\Gamma} \times \exp \left[E / E_{\text {fold }}\right]\right.$, where $E$ is the photon energy, $\Gamma$ and $E_{\text {fold }}$ are the photon index and the folding energy, respectively) modified at lower energies by photoelectric absorption.

The best-fit parameters are $\Gamma=1.6(1), E_{\text {fold }}=30(2) \mathrm{keV}$, the absorption column density $n_{\mathrm{H}}=11(1) \times 10^{22}$ hydrogen atoms per $\mathrm{cm}^{2}$. The uncertainties in parentheses refer to the last digit and are at the $90 \%$ confidence level. The value of $n_{\mathrm{H}}$ is substantially higher than measured in previous observations of the source, including older INTEGRAL measurements $\left(1-3 \times 10^{22} \mathrm{~cm}^{-2}\right.$, e.g. Klochkov et al. 2007; Wilson et al. 2008). We note, however, that studying $n_{\mathrm{H}}$ with JEM-X is generally problematic as the data below $\sim 3 \mathrm{keV}$ are not available. The significance of the measured increase in absorption is therefore questionable.

The data quality does not permit spectroscopy of individual flares. To characterize the spectral behavior of EXO 2030+375 during the flaring episode and compare it with the rest of the outburst, we explored the luminosity-dependence of the source spectrum during the rising (flaring) part and the decay of the outburst. We grouped the individual INTEGRAL pointings according to the measured flux in the $20-80 \mathrm{keV}$ range. For each group we extracted and analyzed the X-ray spectrum using the spectral model described above. The statistics did not allow us to explore the dependence of each individual spectral parameter on flux. We therefore fixed $n_{\mathrm{H}}$ and $E_{\text {fold }}$ to their averaged values $\left(11 \times 10^{22} \mathrm{~cm}^{-2}\right.$ and $30 \mathrm{keV}$, respectively) and explored the photon index $\Gamma$ as a function of flux.

The resulting dependence is shown in Fig. 6 for the rise (left) and decay (right) of the outburst. Higher values of $\Gamma$ correspond to a softer spectrum (see the model description above). The horizontal error bars represent the width of the flux bins. The vertical error bars indicate $1 \sigma$-uncertainties. While the spectrum apparently tends to get harder with increasing flux during the rising part of the outburst, it remains roughly constant during the decay. To quantify this behavior, we performed linear fits to the data points and calculated the slopes with the corresponding uncertainties. The slope is $(-9.0 \pm 3.7) \times 10^{-3}(\mathrm{cts} / \mathrm{s})^{-1}$ in the rising part and $(2.8 \pm 4.2) \times 10^{-3}(\mathrm{cts} / \mathrm{s})^{-1}$ in the decay of the outburst (uncertainties at $1 \sigma$ confidence level).

\section{Discussion}

A direct comparison of the flares presented here with those observed with EXOSAT in 1985 revealed significant similarity. The peak fluxes and the relative amplitude of the flares are roughly the same in both episodes. Also the average shape - fast rise/slow decay - is similar in the two cases. The mean period 
is, however, different: $\sim 4 \mathrm{~h}$ for the EXOSAT flares and $\sim 7 \mathrm{~h}$ for the INTEGRAL ones. The location of the EXOSAT flares with respect to the nearest "normal" outburst is difficult to reconstruct because no monitoring of the source flux (apart from the EXOSAT data themselves) was performed at the time. Since the orbital phase of "normal" outbursts varies significantly with time (Wilson et al. 2002), the extrapolation of the orbital phase ephemeris back to the EXOSAT observations would not resolve the problem.

X-ray flares in accreting pulsars are usually attributed to one of the following mechanisms: (1) instabilities of the accretion flow around/within the magnetospheric boundary (e.g. Moon et al. 2003b; Postnov et al. 2008); (2) highly inhomogeneous stellar wind of the donor star (e.g. Taam et al. 1988; Walter \& Zurita Heras 2007); and (3) nuclear burning at the neutron star surface (e.g. Levine et al. 2000; Brown \& Bildsten 1998). The nuclear burning scenario is, however, difficult to reconcile with a relatively high accretion rate before and after a flare, which would suppress the thermonuclear instability (Bildsten \& Brown 1997). In the case of EXO 2030+375, inhomogeneities of the companion's stellar wind are also unlikely to be a direct cause of the flares for the following reasons. First, the viscous time of the accretion disk that is believed to be present in EXO 2030+375 during normal outbursts (Wilson et al. 2002) and to even survive during quiescence (Hayasaki \& Okazaki 2006), is at least several days, which would smooth out any variations in the mass accretion rate $\dot{M}$ caused by inhomogeneity of the wind shorter than this time. Second, nonuniform stellar wind cannot explain the observed quasi-periodic appearance of the flares. On the other hand, various kinds of magneto-hydrodynamic instabilities at the inner edge of the accretion disk may easily lead to oscillations in the mass flow towards the polar caps of the neutron star (Apparao 1991; Postnov et al. 2008; D’ Angelo \& Spruit 2010), leading to the observed flaring activity. For example, D'Angelo \& Spruit (2010) have illustrated that when the magnetospheric radius $r_{\mathrm{m}}$ (where magnetic field of the neutron star truncates the accretion disk) is larger but close to the corotation radius $r_{\mathrm{c}}$ (where the Keplerian frequency is equal to the accretor's spin frequency), matter in the inner regions of the disk will pile up leading to an increase in the local gas pressure and, therefore, a decrease in $r_{\mathrm{m}}$. When $r_{\mathrm{m}}$ crosses $r_{\mathrm{c}}$, the accumulated reservoir of gas is accreted by the neutron star and the cycle repeats.

To assess the applicability of this scenario to the flares observed in EXO 2030+375, we estimated the expected characteristic time scale of $\dot{M}$-oscillations. Without going into physical details of the disk-field coupling at $r_{\mathrm{m}}$ one would generally expect that the oscillations in $\dot{M}$ take place on the time scale close to the local viscous time at $r_{\mathrm{m}} \sim r_{\mathrm{c}}$. Following the standard $\alpha$-viscosity prescription (Shakura \& Sunyaev 1973), this time can be estimated as $\tau_{\mathrm{c}} \sim r_{\mathrm{c}}^{2} / v\left(r_{\mathrm{c}}\right) \sim 1 /\left[\Omega \alpha(H / R)^{2}\right]$, where $v\left(r_{\mathrm{c}}\right)$ is the viscosity at $r_{\mathrm{c}}, \Omega$ is the spin frequency of the neutron star, and $(H / R)$ the semithickness of the accretion disk. Using "standard" values of $\alpha=0.1, H / R=0.05$, and the known pulse period $P \simeq 40 \mathrm{~s}$, one gets $\tau_{\mathrm{c}} \sim 7 \mathrm{~h}$, i.e. of several hours, as was observed.

The averaged profile of the flares is characterized by a steep rise and a slower decay (Fig. 4), which is very similar to the flares observed with EXOSAT in 1985 (Fig. 2 of Parmar et al. 1989). According to the authors, such a shape suggests a "draining reservoir" that is in line with the picture described above (matter piling up on the inner edge of the disk).

The observed difference in the spectral behavior between the flaring part and the rest of the outburst (Sect.4) suggests different configurations of the region where matter couples to the field lines. Such a difference is indeed expected if the flares are caused by the oscillating inner edge of the accretion disk. In this case, matter from the oscillating inner disk rim would couple to different dipole field lines of the neutron star (and follow them) compared to the decay part of the outburst where the configuration of the inner disk rim is presumably stable.

The difference in the mean period of flares in the INTEGRAL and EXOSAT observations can also be understood in the described picture. The period must depend on the mass transfer rate through the accretion disk, i.e. time needed to refill the reservoir. This rate could be different between the EXOSAT and INTEGRAL observations due to, e.g., changes in the state of the Be-disk. In Fig. 2 one might also notice some shortening of the the flare separation time as the flux increases (although this behavior is difficult to quantify with the available statistics). Such behavior, if real, might reflect shortening of the reservoir refill time as the mass transfer rate increases towards the maximum of the outburst.

Thus, we argue that the observational appearance of the flares in EXO 2030+375 suggests that the instability of the inner disk edge (pile-up/draining of matter) is the most probable cause of the flares.

It is important to note that the rarity of the detected flaring episodes (even considering the relatively sparse observational coverage) means that the range of physical conditions needed to initiate flares could be very narrow, which would lead to the serendipitous character of the phenomenon.

Acknowledgements. The work was supported by the Carl-Zeiss-Stiftung and by DLR grant BA5027. This research is based on observations with INTEGRAL, an ESA project with instruments and science data centre funded by ESA member states. The authors thank the anonymous referee for useful suggestions.

\section{References}

Angelini, L., White, N. E., \& Stella, L. 1991, ApJ, 371, 332 Apparao, K. M. V. 1991, ApJ, 375, 701

Bildsten, L., \& Brown, E. F. 1997, ApJ, 477, 897

Brown, E. F., \& Bildsten, L. 1998, ApJ, 496, 915

Caballero, I., Santangelo, A., Kretschmar, P., et al. 2008, A\&A, 480, L17 Camero Arranz, A., Wilson, C. A., Connell, P., et al. 2005, A\&A, 441, 261 Courvoisier, T. J.-L., Walter, R., Beckmann, V., et al. 2003, A\&A, 411, L53 D’Angelo, C. R., \& Spruit, H. C. 2010, MNRAS, 406, 1208 Finger, M. H., Bildsten, L., Chakrabarty, D., et al. 1999, ApJ, 517, 449 Fürst, F., Kreykenbohm, I., Pottschmidt, K., et al. 2010, A\&A, 519, A37 Hayasaki, K., \& Okazaki, A. T. 2006, MNRAS, 372, 1140

Klochkov, D., Horns, D., Santangelo, A., et al. 2007, A\&A, 464, L45

Kreykenbohm, I., Wilms, J., Kretschmar, P., et al. 2008, A\&A, 492, 511 Lebrun, F., Leray, J. P., Lavocat, P., et al. 2003, A\&A, 411, L141 Levine, A. M., Rappaport, S. A., \& Zojcheski, G. 2000, ApJ, 541, 194 Liu, Q. Z., van Paradijs, J., \& van den Heuvel, E. P. J. 2006, A\&A, 455, 1165 Lund, N., Budtz-Jørgensen, C., Westergaard, N. J., et al. 2003, A\&A, 411, L231 Moon, D.-S., Eikenberry, S. S., \& Wasserman, I. M. 2003a, ApJ, 582, L91 Moon, D.-S., Eikenberry, S. S., \& Wasserman, I. M. 2003b, ApJ, 586, 1280 Nandra, K., George, I. M., Mushotzky, R. F., et al. 1997, ApJ, 476, 70

Parmar, A. N., White, N. E., Stella, L., Izzo, C., \& Ferri, P. 1989, ApJ, 338, 359 Postnov, K., Staubert, R., Santangelo, A., et al. 2008, A\&A, 480, L21 Reig, P. 2011, Ap\&SS, 332, 1

Reig, P., \& Coe, M. J. 1998, MNRAS, 294, 118

Reig, P., Belloni, T., Israel, G. L., et al. 2008, A\&A, 485, 797

Shakura, N. I., \& Sunyaev, R. A. 1973, A\&A, 24, 337

Sidoli, L. 2011, Adv. Space Res., 48, 88

Staubert, R., Klochkov, D., \& Wilms, J. 2009, A\&A, 500, 883

Taam, R. E., Brown, D. A., \& Fryxell, B. A. 1988, ApJ, 331, L117

Ubertini, P., Lebrun, F., Di Cocco, G., et al. 2003, A\&A, 411, L131

Vedrenne, G., Roques, J.-P., Schönfelder, V., et al. 2003, A\&A, 411, L63

Walter, R., \& Zurita Heras, J. 2007, A\&A, 476, 335

Wilson, C. A., Finger, M. H., Coe, M. J., et al. 2002, ApJ, 570, 287

Wilson, C. A., Finger, M. H., \& Camero-Arranz, A. 2008, ApJ, 678, 1263

Winkler, C., Courvoisier, T. J.-L., Di Cocco, G., et al. 2003, A\&A, 411, L1 